\title{
Dysfunctional high-density lipoprotein
}

\author{
Hong Feng and Xiang-An Li \\ Kentucky Pediatric Research Institute, Department of Pediatrics, University of Kentucky Medical \\ Center, Lexington, Kentucky, USA
}

\section{Abstract}

Purpose of review—To address the progress of the investigation on dysfunctional high-density lipoprotein (HDL).

Recent findings-HDL is generally considered to be an independent protective factor against cardiovascular disease. However, emerging evidence indicates that HDL can be modified under certain circumstances and lose its protective effect or even become atherogenic. The underlying mechanisms responsible for generating the dysfunctional HDL and the chemical and structural changes of HDL remain largely unknown. Recent studies focus on the role of myeloperoxidase in generating oxidants as participants in rendering HDL dysfunctional in vivo. Myeloperoxidase modifies HDL in humans by oxidation of specific amino acid residues in apolipoprotein A-I, which impairs cholesterol efflux through ATP-binding cassette transporter A1 and contributes to atherogenesis.

Summary-HDL may not always be atheroprotective and can be atherogenic paradoxically under certain conditions. The mechanisms responsible for generating the dysfunctional HDL remain largely unknown. Recent data suggest that myeloperoxidase-associated modification of HDL may be one of the mechanisms. Further studies are needed to investigate the in-vivo mechanisms of HDL modification and identify therapeutic approaches aiming at controlling HDL modification.

\section{Keywords}

apolipoprotein A-I; cardiovascular disease; high-density lipoprotein; lipoprotein; myeloperoxidase

\section{Introduction}

It is well established that high-density lipoprotein cholesterol (HDL-C) concentration in the blood is independently and inversely associated with an increased risk of cardiovascular disease (CVD) $[1 \bullet, 2]$. However, it is apparent that many patients with 'normal' or even 'elevated' plasma HDL experience clinical events [3]. Furthermore, the recent clinical trial ILLUMINATE, which targets to increase plasma HDL levels with a new selective cholesteryl ester transfer protein (CETP) inhibitor, Torcetrapib, was prematurely terminated because of an increase in all-cause mortality despite an increase in HDL-C levels. These disappointing results suggest that HDL may not always be atheroprotective $[4 \bullet \cdot, 5 \bullet$, and in some conditions, it paradoxically enhances the process of atherosclerosis. 


\section{Structure of 'normal' high-density lipoprotein}

HDLs are a heterogeneous class of lipoproteins. They vary in diameter, density, and composition [6]. Generally speaking, HDLs are organized complexes of lipids (cholesterol, cholesteryl esters, triglycerides, and a variety of phospholipids) and proteins (apolipoproteins and enzymes). The surface of these particles is composed of the charged head groups of phospholipids (phosphatidylcholine, phosphatidylserine, cardiolipin, and phosphatidylethanolamine) and amino acids of proteins that project into the aqueous phase. The particle core is a reservoir of hydrophobic lipid (triglycerides and cholesteryl ester) sequestered from the aqueous phase. Analytic ultracentrifugation subdivides HDL into $\mathrm{HDL}_{2}$ (large, buoyant, and lipid enriched; density $=1.063-1.125 \mathrm{~g} / \mathrm{ml}$ ) and $\mathrm{HDL}_{3}$ (small and dense; density $=1.125-1.210 \mathrm{~g} / \mathrm{ml}$ ) with diameters ranging from 70 to $120 \AA$.

Apolipoprotein A-I (apoA-I) comprises roughly 70\% of the HDL mass and apoA-II another 15-20\%. The remainders are made up of amphipathic proteins including the apoCs, apoE, apoD, apoM, apoA-II, paraoxonase (PON), serum amyloid A (SAA), and many other proteins. Early study [7] reported that serum amyloid P (SAP) binds to HDL in a calciumdependent manner, indicating that some of the HDL components may not be present in isolated HDL due to use of EDTA in isolation processes. Recently, Vaisar et al. [8・•] and Reilly and Tall [9•] used shortgun proteomics to investigate the composition of HDL isolated from participants and identified multiple complement-regulatory proteins, as well as a diverse array of distinct serpins with serine-type endopeptidase inhibitor activity and many acute phase response proteins. These lower abundance proteins are not present on all HDL particles and may actually be sequestered on compositionally distinct particles within the density class. These amphipathic proteins form stable micellar complexes with phospholipids, cholesterol, triglycerides, and cholesteryl esters.

HDLs are metabolically active molecules and constantly remodel and interconvert among their various forms by the activity of a number of enzymes [10]. As a result of qualitative and quantitative differences in lipid, protein, and enzyme content, HDL particles are multishaped molecules with varying density, fluidity, charge, and antigenicity. For example, in addition to phosphatidylcholine, the surface of HDL contains other phospholipids such as sphingomyelin, phosphatidylserine, phosphatidylinositol, and phosphatidylethanolamine $[11,12]$. There is also considerable variation in the length and unsaturation of HDL phosphatidylcholine acyl chains, as well as the effects on the inhibition of vascular cell adhesion molecule 1 (VCAM-1) [13]. As another example, apoA-I, the predominant protein of HDL, either in its lipid-free form or as a component of different HDL species, can bind to fibroblasts and macrophages and promote extracellular lipid translocation within blood vessel walls. As demonstrated by both the AFCAPS/TexCAPS Trial [14] and the European Concerted Action on Thrombosis and Disabilities (ECAT)-Angina Pectoris Study [15], as serum levels of apoA-I progressively increase, the risk for coronary events decreases. Thus, the modification of apoA-I may readily impair the atheroprotective function of HDL. So, both protein and phospholipid content can affect the functionality of HDL.

\section{Biological functions of 'normal' high-density lipoprotein}

The cardiovascular protective effects of HDL have been mainly attributed to its role in reverse cholesterol transport. However, a number of pleiotropic atheroprotective effects of HDL are emerging, including antioxidative, anti-inflammatory, antiapoptotic, antithrombotic properties, and so on. 


\section{High-density lipoprotein and reverse cholesterol transport}

The atheroprotective effect of HDL is often explained by the ability of HDL to remove cholesterol from the periphery for delivery to the liver and excretion into the bile, a process named reverse cholesterol transport [16]. As shown in Fig. 1, lipid-free apoA-I is produced in the intestine or liver or shed from the surface of triglyceride-rich lipoproteins during lipolysis. These particles initiate efflux of phospholipids and cholesterol from cell membranes [17]. This process is facilitated by the ATP-binding cassette transporter A1 (ABCA1) that moves cellular lipids across the bilayer in a process requiring hydrolysis of ATP [18]. As ABCA1 does not bind cholesterol [19], it is believed to induce translocation of phospholipids to lipid-poor apoA-I, thus triggering cholesterol efflux to the apoA-Iphospholipids complex. Of note, patients with Tangier disease present a severe deficiency in plasma HDL because of the dysfunctional ABCA1 [20]. Cholesterol in nascent discoidal HDL is then esterified by the action of lecithin-cholesterol acyltransferase (LCAT). Cholesteryl esters readily move to the core of HDL particles, producing a steady gradient of free cholesterol and enabling HDL to further accept cholesterol from various donors. The reciprocal exchange of cholesteryl ester for triglycerides mediated by CETP moves the bulk of the cholesteryl esters to apoB-containing lipoproteins, which are eventually cleared by the liver. At the same time, HDL becomes enriched with triglycerides, which are substrates for CETP and hepatic lipase. The concerted action of CETP-mediated cholesteryl ester transfer and hepatic lipase-mediated hydrolysis of triglycerides and phospholipids leads to smaller HDL particles that are the preferred binding partners for scavenger receptor B type I (SR$\mathrm{BI}$ ), the major HDL receptor in hepatic cells. SR-BI mediates the selective uptake of cholesteryl esters from HDL, resulting in the removal of cholesterol from circulation. Lipidfree apolipoproteins or lipid-poor pre $\beta$-HDL is also formed during the remodeling by phospholipids transfer protein (PLTP), CETP, and hepatic lipase. The cells comprising vessel walls are unable to catabolize cholesterol [21]. Consequently, if reverse cholesterol transport is impaired, more cholesterol accumulates within the sub-endothelial space, leading to progressive atherosclerotic plaque development [17].

\section{Atheroprotective effects beyond reverse cholesterol transport}

In addition to its role in reverse cholesterol transport, HDL shows many other protective properties towards atherosclerosis. HDL inhibits the chemotaxis of monocytes [22,23], prevents endothelial dysfunction and apoptosis [24], prohibits low-density lipoprotein (LDL) oxidation [25,26], and stimulates the proliferation of endothelial cells and smooth muscle cells $[27,28]$. These anti-inflammatory, antioxidative, antiaggregatory, anticoagulant, and pro-fibrinolytic activities are exerted by different components of HDL, namely apolipoproteins, enzymes, and even specific phospholipids. This complexity further emphasizes that changes in the functionality of HDL rather than changes in plasma HDL-C levels determine the antiatherogenicity of therapeutic alterations of HDL metabolism.

Recent researches [29] focused on the multiple anti-inflammatory effects of HDL. The oxidized phospholipids within LDL stimulate arterial wall production of monocyte chemoattractant protein (MCP) 1, which increases recruitment of mononuclear cells and the expression of cellular adhesion molecules that promote leukocyte interaction with endothelial cells and entry into atheroma [30]. By limiting LDL oxidation, HDL thus plays a key anti-inflammatory role in slowing atherogenesis. However, in the setting of acute phase response and systemic inflammation, the anti-inflammatory character of HDL can markedly diminish, even to the point where it becomes proinflammatory [31,32].

Inhibition of LDL oxidation by HDL is usually attributed to the high content of antioxidants in this lipoprotein, antioxidative properties of apoA-I, and the association with HDL of 
several enzymes, such as PON, platelet activating factor acetylhydrolase (PAF-AH), and glutathione peroxidase (GPX), which prevent LDL oxidation and/or contribute to the degradation of bioactive products that are formed during oxidation. The ability of HDL to act as an antioxidant, at the same time, renders HDL itself more susceptible to oxidation. The delicate relation between the modification and the impairment of HDL function probably plays a role in determining the antiatherogenic potential of HDL.

A number of studies [33-36] demonstrated that HDL binding to SR-BI stimulates endothelial nitric oxide synthase (eNOS) activity and enhances endothelium-dependent and nitric oxide-dependent relaxation in the aorta. Moreover, HDL prevents ox-LDL-induced eNOS inactivation by preserving the cholesterol concentration in caveolae, thereby maintaining the subcellular location of eNOS [37]. HDL can also suppress SR-BI-induced apoptosis [38]. In these ways, HDL may be critical in atheroprotective properties.

\section{Dysfunctional high-density lipoprotein as an atherogenic particle}

Although epidemiological studies have consistently demonstrated an inverse association between plasma HDL, as well as apoA-I concentration, and the risk of myocardial infarction, a subset of patients with high plasma HDL concentrations have enhanced rather than reduced atherosclerosis [39]. It has been proposed that modification of HDL may lead to changes in its antiatherogenic properties or even result in an actual promotion of atherogenic events $[29,40 \bullet]$. Meanwhile, when an independent safety monitoring committee in ILLUMINATE revealed an unfavorable imbalance in mortality and revascularizations in the Torcetrapib treatment study in early December 2006 [41•], the scientific community was shocked. Despite the significant increase in HDL level with the treatment of CETP inhibitor, the HDL particles may lose their normal constructions and protective effects. It has been speculated that CETP inhibition by Torcetrapib produced dysfunctional HDLs, leading to impaired reverse cholesterol transport.

It is of interest that HDL recovered from different participants often exhibits marked heterogeneity in its in-vitro functional properties. Several methods of identifying these processes have been described, including the monocyte chemotaxis assay (MCA) and a cellfree assay (CFA) $[42,43 \bullet]$, which measures HDL's ability to alter LDL-induced chemotaxis in a human artery wall coculture and alter oxidized phospholipid-induced fluorescence in a cell-free system, respectively. Using these methods, Ansell et al. [29] examined the characteristics of HDL sampled from patients who developed coronary heart disease (CHD) despite very high HDL cholesterol levels (i.e., $\mathrm{HDL} \geq 84 \mathrm{mg} / \mathrm{dl}$ ). The difference in the antiinflammatory potential of HDL was marked: those individuals who had CHD despite supernormal levels of HDL cholesterol had uniformly proinflammatory MCA and CFA results. They proposed that in setting of both vascular and nonvascular systemic inflammation, including CHD, diabetes, surgery, and influenza infection, the atheroprotective effects of HDL can markedly diminish, even to the point where it becomes proinflammatory. Several similar observations are reported. For example, Gowri et al. [44] reported the decreased protection against LDL oxidation by HDL from poorly controlled diabetic patients, due to the abnormal composition of HDL, and White et al. [45•] demonstrated that, in contrast to promoting eNOS activity, HDL from diabetic patients actually inhibits eNOS activity because of the abnormally high level of myristic acid in HDL. A number of studies [46-48] showed the functional changes of HDL during acute phase responses in humans, rabbits, and mice. All these studies indicate that the dysfunctional form of HDL is associated with alterations in lipid or protein content of circulating HDL. 
Several animal models associated with dysfunctional HDL were established. Berard et al. [49] developed a transgenic mouse model overexpressing human LCAT, the major enzyme promoting the esterification of free cholesterol to cholesteryl ester and packaging cholesterol into the core of HDL. The transgenic mice have elevated HDL and increased diet-induced atherosclerosis, and the observation shows the abnormal HDLs in both composition and function, and corresponding ineffective transport of HDL-C to the liver and impaired reverse cholesterol transport. Using SR-BI-null mice, numerous studies [33,36,50•] demonstrate that despite a marked increase in HDL concentration, the mice developed enhanced atherosclerosis. These genetically manipulated mouse models provide in-vivo evidence for dysfunctional HDL as a potential mechanism leading to increased atherosclerosis in the presence of high plasma HDL levels.

Evidence for the presence of modified HDL in atherosclerotic tissues has also been reported recently. Artola et al. [51] showed the presence of cross-linked apolipoproteins in the absence of elevated lipid peroxidation products in HDL isolated from hypercholesterolemic chickens. Furthermore, by using histochemical analysis and immunoblot [52,53], these modified lipoproteins were detected in atheromatous plaques of the abdominal aorta [54] and in the sera from patients with chronic renal failure [55]. More recently, two independent groups have shown that chlorotyrosine (a specific product of $\mathrm{HOCl}$ ) modified HDL (ClTyrHDL) is present in human atherosclerotic tissue and human plasma [56,57]. The same groups have also shown that plasma ClTyr-HDL levels are increased in patients with CVD, suggesting that circulating levels of modified HDL represent a unique marker for clinically significant atherosclerotic disease. Also, nitrotyrosine-modified HDL $\left(\mathrm{NO}_{2} \mathrm{Tyr}-\mathrm{HDL}\right)$ levels are increased in patients with CVD [57]. These observations suggest that dysfunctional HDL can be generated in vivo and promote atherosclerosis.

\section{High-density lipoprotein modification}

Accumulating data suggest that HDL can easily be modified and lose its antiatherogenic activities through multiple mechanisms. Based on the nature of modification, we classify the modification into three types (Fig. 2): spontaneous oxidative modification [58-60], due to the presence of free metal ions and free radicals in the atherosclerotic plaques, similar to the oxidation of LDL; enzyme-induced modification, including myeloperoxidase (MPO), chymase-tryptase, matrix metalloproteinases (MMPs), PMN-associated enzyme, endothelial lipase, and so on [61-66]. These enzymes can degrade or oxidize apolipoproteins without significant changes in lipid moiety, or alternatively induce apolipoprotein cross-linking and lipid oxidation; metabolic modification, such as glycation $[45 \bullet, 67,68]$ that occurs under hyperglycaemic conditions, and acute phase reactants-induced modification during inflammation and so on $[7,69,70]$.

\section{Myeloperoxidase-induced dysfunctional high-density lipoprotein}

Recently, MPO has been focused on to explain the mechanisms of generating dysfunctional HDL in vivo. MPO is an important member of self-defense systemthat catalyzes the generation of highly reactive oxidants, including hypochlorous acid $(\mathrm{HOCl})$ and peroxynitrite. These powerful oxidants are used to kill microbial pathogens [71]. However, they can also oxidize other molecules due to their high reactivity. Important insights into the mechanisms that oxidize HDL in human artery wall have come from the mass spectrometric (MS) detection of oxidized amino acids that result from specific reaction pathways. Zheng et al. [57] reported the dramatic selective enrichment in $\mathrm{NO}_{2} \mathrm{Tyr}$ and ClTyr contents within apoA-I recovered from serum and human atherosclerotic lesions, and analysis of serum from sequential participants demonstrates that the $\mathrm{NO}_{2} \mathrm{Tyr}$ and $\mathrm{ClTyr}$ contents of apoA-I are markedly higher in individuals with CVD. These findings support a direct role for nitric 
oxide-derived oxidants and MPO-catalyzed halogenation as oxidation pathways relevant to apoA-I oxidation in vivo, because $\mathrm{NO}_{2} \mathrm{Tyr}$ and $\mathrm{ClTyr}$ serve as selective molecular markers of posttranslational protein modification by these respective pathways [72,73].MPO is the only known enzyme in mammals capable of generating chlorinating oxidants. Thus, Zheng et al. [57] demonstrated that apoA-I serves as a selective target of oxidation both within the systemic circulation and in human atherosclerotic plaque. Further independent confirmation of the role of apoA-I as a target for MPO-catalyzed oxidation has been reported by Bergt $e t$ al. [56] recently.

MPO as a likely mechanism for oxidative modification of apoA-I in vivo is apparently facilitated by MPO binding to apoA-I, as revealed by cross-immunoprecipitation studies in plasma, recovery of MPO within HDL-like particles isolated from human atheroma, and identification of a probable contact site between the apoA-I moiety of HDL and MPO. A specific binding site for MPO on HDL within the helix 8 domain of apoA-I has been identified through biophysical studies employing hydrogen deuterium exchange coupled with tandem mass spectrometry [74]. Site-specific liquid chromatography-mass spectrometry quantitative analyses demonstrated that the favored modification sites following exposure to MPO-generated oxidants are Tyr-166 and Tyr-192. Parallel functional studies demonstrated that site-specific modifications of apoA-I by MPO are associated with impaired lipid binding and ABCA1-dependent cholesterol efflux activity, providing a molecular mechanism that likely contributes to the clinical link between MPO levels and CVD risk. The corresponding functional alterations in apoA-I cholesterol efflux activity, combined with clinical observations linking chemical signatures of apoA-I nitration and chlorination with increased risk for cardiovascular disease, strongly support the concept that MPO-mediated oxidation of HDL generates a dysfunctional lipoprotein capable of contributing to the atherosclerotic process (Fig. 3). Independent groups have now demonstrated that systemic levels of apoA-I modified by nitration or chlorination are correlated with CVD prevalence [56,57,75], suggesting the potential of these modified forms of lipoprotein to serve as markers of cardiovascular risk prediction.

Nevertheless, the in-vivo mechanisms involved in HDL modification are largely unknown. A better knowledge of these pathways may provide possible therapeutic target aiming at reducing HDL modification.

\section{Conclusion}

Recent studies suggest that under some conditions, HDL can readily be modified to lose its atheroprotective properties and become dysfunctional or even atherogenic. Therefore, we should not only evaluate HDL level but also measure HDL function while we predict the risk of CVD. Future studies are clearly warranted to investigate the in-vivo mechanisms of HDL modification in relation to CVD and to identify nutritional and therapeutic approaches aiming at controlling HDL modification.

\section{Acknowledgments}

This work was supported by grants from American Heart Association (0530241N), NIH (R01GM085231), and Children Miracle Network.

\section{References and recommended reading}

Papers of particular interest, published within the annual period of review, have been highlighted as:

- of special interest 
$\bullet$ of outstanding interest

Additional references related to this topic can also be found in the Current World Literature section in this issue (p. 196).

1. Majeed F, Miller M. Low high-density lipoprotein cholesterol: an important consideration in coronary heart disease risk assessment. Curr Opin Endocrinol Diabetes Obes. 2008; 15:175-181. [PubMed: 18316954] This article summarizes the recent view on the positive function of HDL.

2. Gordon TCW, Hjortland MC, et al. High density lipoprotein as a protective factor against coronary heart disease. The Framingham Study. Am J Med. 1977; 62:707-714. [PubMed: 193398]

3. Pearson H. When good cholesterol turns bad. Nature. 2006; 444:794-795. [PubMed: 17167436]

4. Nissen SE, Tardif JC, Nicholls SJ, et al. Effect of torcetrapib on the progression of coronary atherosclerosis. N Engl J Med. 2007; 356:1304-1316. [PubMed: 17387129] This article demonstrates the shocking result of the recent clinical trial ILLUMINATE on the selective CETP inhibitor Torcetrapib and proposes the likely mechanisms.

5. Barkowski RS, Frishman WH. HDL metabolism and CETP inhibition. Cardiol Rev. 2008; 16:154162. [PubMed: 18414186] This review summarizes the relationship between HDL metabolism and CETP inhibition, which aims to explain the unexpected result of clinical trial on Torcetrapib.

6. Schaefer EJ, Foster DM, Jenkins LL, et al. The composition and metabolism of high density lipoprotein subfractions. Lipids. 1979; 14:511-522. [PubMed: 222991]

7. Li XA, Yutani C, Shimokado K. Serum amyloid P component associates with high density lipoprotein as well as very low density lipoprotein but not with low density lipoprotein. Biochem Biophys Res Commun. 1998; 244:249-252. [PubMed: 9514915]

8. Vaisar T, Pennathur S, Green PS, et al. Shotgun proteomics implicates protease inhibition and complement activation in the anti-inflammatory properties of HDL. J Clin Invest. 2007; 117:746756. [PubMed: 17332893] The investigators first used proteomics to study the protein content of HDL, and the result further supports the anti-inflammatory properties of HDL.

9. Reilly MP, Tall AR. HDL proteomics: pot of gold or Pandora's box? J Clin Invest. 2007; 117:595598. [PubMed: 17332889] This article points out that the HDL proteomics is in its infancy and the result needs to be confirmed.

10. Toth PP. High-density lipoprotein: epidemiology, metabolism, and antiatherogenic effects. Dis Mon. 2001; 47:369-416. [PubMed: 11571478]

11. Bagdade JD, Buchanan WF, Pollare T, Lithell H. Abnormal lipoprotein phospholipid composition in patients with essential hypertension. Atherosclerosis. 1995; 117:209-215. [PubMed: 8801866]

12. Davidson WS, Sparks DL, Lund-Katz S, Phillips MC. The molecular basis for the difference in charge between prebeta- and alpha-migrating high density lipoproteins. J Biol Chem. 1994; 269:8959-8965. [PubMed: 8132633]

13. Baker PW, Rye KA, Gamble JR, et al. Phospholipid composition of reconstituted high density lipoproteins influences their ability to inhibit endothelial cell adhesion molecule expression. J Lipid Res. 2000; 41:1261-1267. [PubMed: 10946014]

14. Sniderman AD, Bergeron J, Frohlich J. Apolipoprotein B versus lipoprotein lipids: vital lessons from the AFCAPS/TexCAPS trial. CMAJ. 2001; 164:44-47. [PubMed: 11209748]

15. Bolibar I, von Eckardstein A, Assmann G, Thompson S. Short-term prognostic value of lipid measurements in patients with angina pectoris. The ECAT Angina Pectoris Study Group: European Concerted Action on Thrombosis and Disabilities. Thromb Haemost. 2000; 84:955-960. [PubMed: 11154140]

16. Silver DL, Jiang XC, Arai T, et al. Receptors and lipid transfer proteins in HDL metabolism. Ann N Y Acad Sci. 2000; 902:103-111. discussion 111-112. [PubMed: 10865830]

17. von Eckardstein A, Nofer JR, Assmann G. High density lipoproteins and arteriosclerosis. Role of cholesterol efflux and reverse cholesterol transport. Arterioscler Thromb Vasc Biol. 2001; 21:1327. [PubMed: 11145929]

18. Gura T. Gene linked to faulty cholesterol transport. Science. 1999; 285:814-815. [PubMed: 10454927] 
19. Wang N, Silver DL, Thiele C, Tall AR. ATP-binding cassette transporter A1 (ABCA1) functions as a cholesterol efflux regulatory protein. J Biol Chem. 2001; 276:23742-23747. [PubMed: 11309399]

20. Rust S, Rosier M, Funke H, et al. Tangier disease is caused by mutations in the gene encoding ATP-binding cassette transporter 1. Nat Genet. 1999; 22:352-355. [PubMed: 10431238]

21. Yokoyama S. Release of cellular cholesterol: molecular mechanism for cholesterol homeostasis in cells and in the body. Biochim Biophys Acta. 2000; 1529:231-244. [PubMed: 11111092]

22. Poston RN, Haskard DO, Coucher JR, et al. Expression of intercellular adhesion molecule-1 in atherosclerotic plaques. Am J Pathol. 1992; 140:665-673. [PubMed: 1372160]

23. van der Wal AC, Das PK, Tigges AJ, Becker AE. Adhesion molecules on the endothelium and mononuclear cells in human atherosclerotic lesions. Am J Pathol. 1992; 141:1427-1433. [PubMed: 1281621]

24. Toborek M, Kaiser S. Endothelial cell functions. Relationship to atherogenesis. Basic Res Cardiol. 1999; 94:295-314. [PubMed: 10543305]

25. Hessler JR, Robertson AL Jr, Chisolm GM 3rd. LDL-induced cytotoxicity and its inhibition by HDL in human vascular smooth muscle and endothelial cells in culture. Atherosclerosis. 1979; 32:213-229. [PubMed: 223585]

26. Parthasarathy S, Barnett J, Fong LG. High-density lipoprotein inhibits the oxidative modification of low-density lipoprotein. Biochim Biophys Acta. 1990; 1044:275-283. [PubMed: 2344447]

27. Ross R, Glomset JA. Atherosclerosis and the arterial smooth muscle cell: proliferation of smooth muscle is a key event in the genesis of the lesions of atherosclerosis. Science. 1973; 180:13321339. [PubMed: 4350926]

28. Libby P, Miao P, Ordovas JM, Schaefer EJ. Lipoproteins increase growth of mitogen-stimulated arterial smooth muscle cells. J Cell Physiol. 1985; 124:1-8. [PubMed: 3930513]

29. Ansell BJ, Navab M, Hama S, et al. Inflammatory/antiinflammatory properties of high-density lipoprotein distinguish patients from control subjects better than high-density lipoprotein cholesterol levels and are favorably affected by simvastatin treatment. Circulation. 2003; 108:2751-2756. [PubMed: 14638544]

30. Navab M, Ananthramaiah GM, Reddy ST, et al. The oxidation hypothesis of atherogenesis: the role of oxidized phospholipids and HDL. J Lipid Res. 2004; 45:993-1007. [PubMed: 15060092]

31. Ansell BJ, Fonarow GC, Fogelman AM. High-density lipoprotein: is it always atheroprotective? Curr Atheroscler Rep. 2006; 8:405-411. [PubMed: 16901411]

32. Navab M, Ananthramaiah GM, Reddy ST, et al. The double jeopardy of HDL. Ann Med. 2005; 37:173-178. [PubMed: 16019715]

33. Li XA, Titlow WB, Jackson BA, et al. High density lipoprotein binding to scavenger receptor, Class B, type I activates endothelial nitric-oxide synthase in a ceramide-dependent manner. J Biol Chem. 2002; 277:11058-11063. [PubMed: 11792700]

34. Yuhanna IS, Zhu Y, Cox BE, et al. High-density lipoprotein binding to scavenger receptor-BI activates endothelial nitric oxide synthase. Nat Med. 2001; 7:853-857. [PubMed: 11433352]

35. Mineo C, Yuhanna IS, Quon MJ, Shaul PW. High density lipoprotein-induced endothelial nitricoxide synthase activation is mediated by Akt and MAP kinases. J Biol Chem. 2003; 278:91429149. [PubMed: 12511559]

36. Gong M, Wilson M, Kelly T, et al. HDL-associated estradiol stimulates endothelial NO synthase and vasodilation in an SR-BI-dependent manner. J Clin Invest. 2003; 111:1579-1587. [PubMed: 12750408]

37. Uittenbogaard A, Shaul PW, Yuhanna IS, et al. High density lipoprotein prevents oxidized low density lipoprotein-induced inhibition of endothelial nitric-oxide synthase localization and activation in caveolae. J Biol Chem. 2000; 275:11278-11283. [PubMed: 10753938]

38. Li XA, Guo L, Dressman JL, et al. A novel ligand-independent apoptotic pathway induced by scavenger receptor class B, type I and suppressed by endothelial nitric-oxide synthase and high density lipoprotein. J Biol Chem. 2005; 280:19087-19096. [PubMed: 15749707]

39. Kwiterovich PO Jr. The antiatherogenic role of high-density lipoprotein cholesterol. Am J Cardiol. 1998; 82:13Q-21Q. [PubMed: 9671001] 
40. Valiyaveettil M, Kar N, Ashraf MZ, et al. Oxidized high-density lipoprotein inhibits platelet activation and aggregation via scavenger receptor BI. Blood. 2008; 111:1962-1971. [PubMed: 17993610] This study demonstrates the positive aspect of function of oxidized HDL.

41. van der Steeg WA, Holme I, Boekholdt SM, et al. High-density lipoprotein cholesterol, highdensity lipoprotein particle size, and apolipoprotein A-I: significance for cardiovascular risk - the IDEAL and EPIC-Norfolk studies. J Am Coll Cardiol. 2008; 51:634-642. [PubMed: 18261682] This article demonstrates that not only the HDL-C concentration but also the HDL particle size and apoA-I should be considered while assessing the risk of CVD.

42. Navab M, Hama SY, Hough GP, et al. A cell-free assay for detecting HDL that is dysfunctional in preventing the formation of or inactivating oxidized phospholipids. J Lipid Res. 2001; 42:13081317. [PubMed: 11483633]

43. Ansell BJ. Targeting the anti-inflammatory effects of high-density lipoprotein. Am J Cardiol. 2007; 100:n3-n9. [PubMed: 18047850] This article shows the paradoxical proinflammatory effects of dysfunctional HDL in systemic inflammation.

44. Gowri MS, Van der Westhuyzen DR, Bridges SR, Anderson JW. Decreased protection by HDL from poorly controlled type 2 diabetic subjects against LDL oxidation may Be due to the abnormal composition of HDL. Arterioscler Thromb Vasc Biol. 1999; 19:2226-2233. [PubMed: 10479666]

45. White J, Guerin T, Swanson H, et al. Diabetic HDL-associated myristic acid inhibits acetylcholineinduced nitric oxide generation by preventing the association of endothelial nitric oxide synthase with calmodulin. Am J Physiol Cell Physiol. 2008; 294:C295-C305. [PubMed: 17977947] This article illustrates the lipid-associated dysfunctional HDL in diabetes.

46. Ashby D, Gamble J, Vadas M, et al. Lack of effect of serum amyloid A (SAA) on the ability of high-density lipoproteins to inhibit endothelial cell adhesion molecule expression. Atherosclerosis. 2001; 154:113-121. [PubMed: 11137089]

47. Van Lenten BJ, Hama SY, de Beer FC, et al. Anti-inflammatory HDL becomes pro-inflammatory during the acute phase response. Loss of protective effect of HDL against LDL oxidation in aortic wall cell cocultures. J Clin Invest. 1995; 96:2758-2767. [PubMed: 8675645]

48. Van Lenten BJ, Wagner AC, Nayak DP, et al. High-density lipoprotein loses its anti-inflammatory properties during acute influenza a infection. Circulation. 2001; 103:2283-2288. [PubMed: 11342478]

49. Berard AM, Foger B, Remaley A, et al. High plasma HDL concentrations associated with enhanced atherosclerosis in transgenic mice overexpressing lecithin-cholesteryl acyltransferase. Nat Med. 1997; 3:744-749. [PubMed: 9212100]

50. Van Eck M, Hoekstra M, Hildebrand RB, et al. Increased oxidative stress in scavenger receptor BI knockout mice with dysfunctional HDL. Arterioscler Thromb Vasc Biol. 2007; 27:2413-2419. [PubMed: 17717299] This study demonstrates the role of SR-BI and the SR-BI-associated HDL dysfunction.

51. Artola RL, Conde CB, Bagatolli L, et al. High-density lipoprotein from hypercholesterolemic animals has peroxidized lipids and oligomeric apolipoprotein A-I: its putative role in atherogenesis. Biochem Biophys Res Commun. 1997; 239:570-574. [PubMed: 9344872]

52. Nakano T, Nagata A. Immunochemical detection of circulating oxidized highdensity lipoprotein with antioxidized apolipoprotein A-I monoclonal antibody. J Lab Clin Med. 2003; 141:378-384. [PubMed: 12819635]

53. Matsunaga T, Koyama I, Hokari S, Komoda T. Detection of oxidized high-density lipoprotein. J Chromatogr B Anal Technol Biomed Life Sci. 2002; 781:331-343.

54. Nakajima T, Origuchi N, Matsunaga T, et al. Localization of oxidized HDL in atheromatous plaques and oxidized HDL binding sites on human aortic endothelial cells. Ann Clin Biochem. 2000; 37(Pt 2):179-182. [PubMed: 10735361]

55. Tsumura M, Kinouchi T, Ono S, et al. Serum lipid metabolism abnormalities and change in lipoprotein contents in patients with advanced-stage renal disease. Clin Chim Acta. 2001; 314:2737. [PubMed: 11718676]

56. Bergt C, Pennathur S, Fu X, et al. The myeloperoxidase product hypochlorous acid oxidizes HDL in the human artery wall and impairs ABCA1-dependent cholesterol transport. Proc Natl Acad Sci U S A. 2004; 101:13032-13037. [PubMed: 15326314] 
57. Zheng L, Nukuna B, Brennan ML, et al. Apolipoprotein A-I is a selective target for myeloperoxidase-catalyzed oxidation and functional impairment in subjects with cardiovascular disease. J Clin Invest. 2004; 114:529-541. [PubMed: 15314690]

58. Nagano Y, Arai H, Kita T. High density lipoprotein loses its effect to stimulate efflux of cholesterol from foam cells after oxidative modification. Proc Natl Acad Sci U S A. 1991; 88:6457-6461. [PubMed: 1862074]

59. Hurtado I, Fiol C, Gracia V, Caldu P. In vitro oxidised HDL exerts a cytotoxic effect on macrophages. Atherosclerosis. 1996; 125:39-46. [PubMed: 8831925]

60. Lamb DJ, Leake DS. Iron released from transferrin at acidic $\mathrm{pH}$ can catalyse the oxidation of low density lipoprotein. FEBS Lett. 1994; 352:15-18. [PubMed: 7925932]

61. Pussinen PJ, Metso J, Keva R, et al. Plasma phospholipid transfer protein-mediated reactions are impaired by hypochlorite-modification of high density lipoprotein. Int J Biochem Cell Biol. 2003; 35:192-202. [PubMed: 12479869]

62. Bergt C, Reicher H, Malle E, Sattler W. Hypochlorite modification of high density lipoprotein: effects on cholesterol efflux from J774 macrophages. FEBS Lett. 1999; 452:295-300. [PubMed: 10386609]

63. Favari E, Lee M, Calabresi L, et al. Depletion of prebeta-high density lipoprotein by human chymase impairs ATP-binding cassette transporter A1- but not scavenger receptor class B type Imediated lipid efflux to high density lipoprotein. J Biol Chem. 2004; 279:9930-9936. [PubMed: 14701812]

64. Lindstedt L, Saarinen J, Kalkkinen N, et al. Matrix metalloproteinases-3, -7, and -12, but not -9, reduce high density lipoprotein-induced cholesterol efflux from human macrophage foam cells by truncation of the carboxyl terminus of apolipoprotein A-I. Parallel losses of prebeta particles and the high affinity component of efflux. J Biol Chem. 1999; 274:22627-22634. [PubMed: 10428843]

65. Cogny A, Paul JL, Atger V, et al. Structural changes of high-density-lipoprotein apolipoproteins following incubation with human polymorphonuclear cells. Eur J Biochem. 1994; 222:965-973. [PubMed: 8026507]

66. Gauster M, Oskolkova OV, Innerlohinger J, et al. Endothelial lipase-modified high-density lipoprotein exhibits diminished ability to mediate SR-BI (scavenger receptor B type I)-dependent free-cholesterol efflux. Biochem J. 2004; 382:75-82. [PubMed: 15080796]

67. Ferretti G, Bacchetti T, Marchionni C, Dousset N. Effect of nonenzymatic glycation on aluminium-induced lipid peroxidation of human high density lipoproteins (HDL). Nutr Metab Cardiovasc Dis. 2004; 14:358-365. [PubMed: 15853120]

68. Ferretti G, Bacchetti T, Marchionni C, et al. Effect of glycation of high density lipoproteins on their physicochemical properties and on paraoxonase activity. Acta Diabetol. 2001; 38:163-169. [PubMed: 11855794]

69. Coetzee GA, Strachan AF, van der Westhuyzen DR, et al. Serum amyloid A-containing human high density lipoprotein 3. Density, size, and apolipoprotein composition. J Biol Chem. 1986; 261:9644-9651. [PubMed: 3525531]

70. Jaross W, Eckey R, Menschikowski M. Biological effects of secretory phospholipase A(2) group IIA on lipoproteins and in atherogenesis. Eur J Clin Invest. 2002; 32:383-393. [PubMed: 12059982]

71. Carr AC, McCall MR, Frei B. Oxidation of LDL by myeloperoxidase and reactive nitrogen species: reaction pathways and antioxidant protection. Arterioscler Thromb Vasc Biol. 2000; 20:1716-1723. [PubMed: 10894808]

72. Brennan ML, Wu W, Fu X, et al. A tale of two controversies: defining both the role of peroxidases in nitrotyrosine formation in vivo using eosinophil peroxidase and myeloperoxidase-deficient mice, and the nature of peroxidase-generated reactive nitrogen species. J Biol Chem. 2002; 277:17415-17427. [PubMed: 11877405]

73. Hazen SL, Heinecke JW. 3-Chlorotyrosine, a specific marker of myeloperoxidase catalyzed oxidation, is markedly elevated in low density lipoprotein isolated from human atherosclerotic intima. J Clin Invest. 1997; 99:2075-2081. [PubMed: 9151778] 
74. Zheng L, Settle M, Brubaker G, et al. Localization of nitration and chlorination sites on apolipoprotein A-I catalyzed by myeloperoxidase in human atheroma and associated oxidative impairment in ABCA1-dependent cholesterol efflux from macrophages. J Biol Chem. 2005; 280:38-47. [PubMed: 15498770]

75. Pennathur S, Bergt C, Shao B, et al. Human atherosclerotic intima and blood of patients with established coronary artery disease contain high density lipoprotein damaged by reactive nitrogen species. J Biol Chem. 2004; 279:42977-42983. [PubMed: 15292228] 


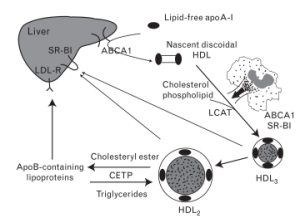

Figure 1. High-density lipoprotein metabolism and reverse cholesterol transport Lipid-free apoA-I is produced in the intestine or liver or shed from the surface of triglyceride-rich lipoproteins during lipolysis. This particle initiates efflux of phospholipids and cholesterol from cell membranes that is facilitated by ABCA1. As the apoA-I adsorbs more lipids, it is converted into a hockey puck-like structure, nascent discoidal high-density lipoprotein (HDL). Cholesterol and phospholipid are esterified by the action of lecithincholesterol acyltransferase (LCAT) and then packed into the core of HDL. In macrophages, ABCA1 mediates cholesterol efflux to apoA-I. As more and more cholesteryl esters are incorporated into the particle, it becomes rounder and progressively larger $\left(\mathrm{HDL}_{3}-\mathrm{HDL}_{2}\right)$. The reciprocal exchange of cholesteryl ester for triglycerides mediated by CETP moves the bulk of the cholesteryl esters to apoB-containing lipoproteins, which are eventually cleared by the liver through low-density lipoprotein (LDL) receptor (LDL-R). Peripheral cholesteryl esters can also be delivered to the liver through the binding of HDL to scavenger receptor B type I (SR-BI) and can also be secreted to bile. 


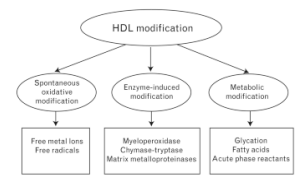

Figure 2. High-density lipoprotein modification

High-density lipoprotein (HDL) modification can be classified into three types based on the nature of modification: spontaneous oxidative modification; enzyme-induced modification; and metabolic modification. 


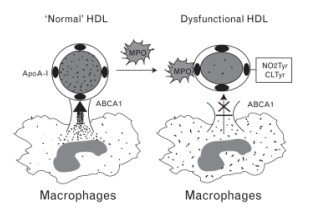

Figure 3. Myeloperoxidase-induced dysfunctional high-density lipoprotein Myeloperoxidase (MPO) site specifically binds to apoA-I and produces reactive oxidative species that are responsible for the nitration and chlorination of tyrosine residues within apoA-I. Consequently, the modified high-density lipoprotein (HDL) impairs the ABCA1dependent cholesterol efflux activity in macrophages. 